Journal of Computer Science 8 (5): 767-774, 2012

ISSN 1549-3636

(C) 2012 Science Publications

\title{
A New Hybrid Approach for Heterogeneous Radio Access Technology Selection
}

\author{
${ }^{1}$ Preethi, J. and ${ }^{2}$ S. Palaniswami \\ ${ }^{1}$ Department of Computer Science and Engineering Anna \\ University of Technology, Coimbatore India \\ ${ }^{2}$ Government College of Engineering, Bargur India
}

\begin{abstract}
Problem statement: The Next generation wireless networks are heterogeneous in nature where different Radio Access Technologies (RAT) coexists in the same coverage area. The user terminal should be able to pick the best access technology such as Wireless Local Area Network (WLAN), Wireless Wide Area Networks (WWAN), the Universal Mobile Telecommunication Systems (UMTS) and the Global System for Mobile Telecommunication (GSM)/Enhanced Data rate for GSM Evolution (EDGE) Radio Access Network (GERAN) at its current location and use the access technology seamlessly for the provision of desired service. The goal is to select the most suitable Radio Access Technology (RAT) for each user. It is important that suitable decision making algorithm should be designed such that all the important characteristics of the system are taken into account when making the radio access selection. Approach: For this reason, this research work proposes a methodology to select the best access network among WLAN and WWAN under the input criteria Signal Strength of both networks, speed of the mobile terminal and network coverage of both networks and Quality of Service (QoS). Hence, in this work, a new hybrid multi objective decision making algorithm employing weighing function and particle swarm optimization is proposed for fuzzy based Radio Access Technology selection of Heterogeneous wireless networks. Results: The end results indicate that the proposed algorithm outperforms better than the existing methods. Conclusion: Inferences from the results indicate that by using the proposed algorithm, best RAT can be selected for the user with a minimal time constraint.
\end{abstract}

Key words: Fuzzy logic controller, multi objective decision making algorithm, weighing function, particle swarm optimization and best access selection

\section{INTRODUCTION}

Over the past twenty years, a number of wireless technologies were developed. The first generation systems are based on analog radio transmission. The Second Generation systems $2 \mathrm{G}$ brought a number of significant advancements over $1 \mathrm{G}$ wireless networks. The second generation systems like TDMA or CDMA systems are completely digital in nature. The Third generation systems like Universal Mobile Telecommunication System (UMTS), CDMA 2000 based on Wireless CDMA technologies is to provide high speed wireless communication to support multimedia, video, data and voice. The wireless transmission has progressed through $1 \mathrm{G}, 2 \mathrm{G}, 2.5 \mathrm{G}$ to $3 \mathrm{G}$ to increase the radio system capacities and per user data rates over $2 \mathrm{G}$, to support IP-based data, voice and multimedia services and at the same time improve interoperability and QoS (Asif, 2007).
It is envisioned that Beyond-3G system will integrate these heterogeneous networks to offer overlapping coverage to mobile users. In turn, the next generation of networks will support multiple technologies, handles the mobility of end users to move through heterogeneous access networks, with the ability to connect to different networks (Hassan and Hassan, 2011). The user terminal has to pick the best access technology such as Wireless Local Area Network (WLAN), the Universal Mobile Telecommunication Systems (UMTS) and the Global System for Mobile Telecommunication (GSM)/Enhanced Data rate for GSM Evolution (EDGE) Radio Access Network (GERAN) at its current location in order to use the technology with the desired service. The goal is to select the most suitable RAT for each user. It is important that suitable decision making algorithm should be designed such that all the important characteristics of the system are taken into account when making the radio access selection.

Corresponding Author:Preethi, J., Department of Computer Science and Engineering, Anna University of Technology,

Coimbatore, Jothipuram Post, Coimbatore, India Tel: 9489362380 
The Key contributions of this work are: This research work proposes an algorithmic approach to select the best access network among WLAN and WWAN under the input criteria Signal Strength of both networks, speed of the mobile terminal and network coverage of both networks and Quality of Service. In this study, a new hybrid multi objective decision making algorithm employing weighing function and Particle swarm optimization is proposed for fuzzy based Radio Access Technology selection of heterogeneous wireless networks.

The rest of the study is organized as follows: Chapter II explains the literature survey on Heterogeneous RAT networks Selection, Chapter III explains about the fuzzy based selection, Chapter IV gives the implementation of proposed work and Chapter V gives the simulation results Chapter VI gives the discussions and finally Chapter VII presents the conclusion.

Related works: Future Wireless Networks does not consist of single radio access network like WWAN, UMTS, but it consists of heterogeneous radio access network that coexist in the same coverage area. The main objective is to decide the best suitable RAT for each call.

A number of RAT selection algorithms are available for initial RAT selection and vertical Handover. References (Sabbagh et al., 2011; Falowo and Chana, 2008) present the good review of RAT selection algorithms.

In Random based RAT selection, when a new call or vertical handover arrives, any of the available RATs will be selected randomly. In Load balancing based RAT selection, the main objective is to uniformly distribute the load among the available RATs in heterogeneous wireless networks (Tolli and Hakalin, 2002; Suleiman et al., 2006; Umbert et al., 2007). In policy based RAT selection, it allocates users to the RAT based on some specific rules specified by the network (Perez-Romero et al., 2005; 2006). Service class based RAT admits calls into particular RAT based on class of service such as voice, video streaming, real time video, web browsing (Zhang, 2005). Service cost based RAT admits incoming call into the least expensive RAT in order to reduce the service cost incurred by the users. Path loss based RAT selection algorithm makes call admission algorithm based on path loss measurements taken in the cells of each RAT Perez-Romero et al., 2006).

In Mobile based RAT selection algorithm (Aleksandar and Janevski, 2011), the mobile terminal measurements from different radio access technology is used for the initial RAT selection. This algorithm uses Particle Swarm Optimization, Fuzzy logic controller, Genetic Algorithm and decision making algorithm for the selection of RAT. The fuzzy neural mechanism is used in (Agusti et al., 2004; 2008) for the selection of best RAT among UMTS, GERAN and WLAN. Thus many RAT selection algorithms are available for the selection of RAT.

Fuzzy inference system: The brief overview of the fuzzy logic and fuzzy controllers are discussed further in this topic.

Fuzzy logic: Fuzzy logic introduced in the year 1965 by Lotfi A.Zadeh, is a mathematical tool for dealing with uncertainty (Ross, 2010). It provides an mechanism for representing linguistic constructs such as "high", "low", "medium", "tall”, In fuzzy systems, the values are indicated by a number ranging from 0 to 1. The membership function for the set maps each element of the set to a membership value between 0 and 1. Generally, Triangular or bell shaped functions are used for representing membership functions.

Fuzzy controllers: Fuzzy logic Control (FLC) is the most active research area in the application of fuzzy set theory, fuzzy reasoning and fuzzy logic. The basic architecture of fuzzy logic controller is shown in Fig. 1.

The principal components of FLC systems as discussed in (Yuhui et al., 1999; Sivanandam and Deepa, 2007) are: a fuzzifier, a fuzzy rule base, an inference engine and a defuzzifier. The fuzzifier converts the crisp quantities into fuzzy quantities. The crisp quantities are represented using membership function. The inference system determines the fuzzy output using fuzzy rules which are in the form of Ifthen rules. The defuzzifier converts the fuzzy output again into crisp output. There are two types of fuzzy systems in fuzzy logic controllers: Mamdami and Sugeno systems. Mamdani's fuzzy inference method is the most commonly seen fuzzy methodology. The Mamdani method finds the centroid of a twodimensional function rather than integrating across the two-dimensional function whereas the Sugeno type systems (Takagi and Sugeno, 1985) use the weighted average of a few data points. Fuzzy logic controllers are cheaper to develop, they cover a wider range of operating conditions and they are more readily customizable in natural language terms (AI-Odienat and Al-Lawama, 2008). The Defuzzification methods are of five types namely: the method of Centroid of area, the method of bisector of area, Mean Of Maximum (MOM), Smallest Of Maximum (SOM) and Largest Of Maximum (LOM). 


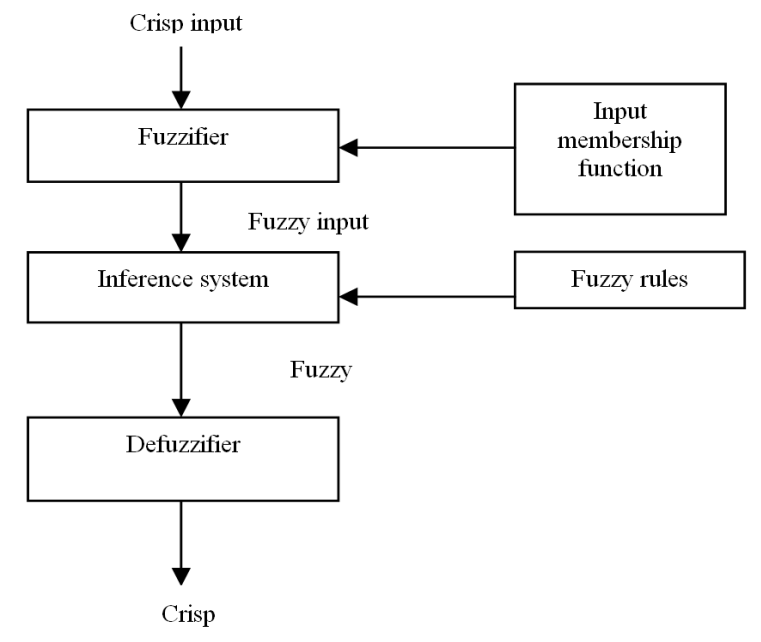

Fig. 1: Basic architecture of fuzzy controller

\section{MATERIALS AND METHODS}

The proposed fuzzy based heterogeneous radio access technology selection using Hybrid Multiobjective decision making algorithm: The authors (Aleksandar and Janevski, 2011) proposed the efficient radio access technology selection that uses the mobile terminal measurements from different radio access technologies. The input parameters considered in (Aleksandar and Janevski, 2011) is received signal strength, cost of the radio access technology, speed of the mobile terminal and QoS like Delay and bit rate. The input parameters are optimized using fuzzy logic controllers, genetic algorithm, particle swarm optimization and multi criteria decision making algorithm for decision making of RAT. The authors (Alkhawlani and Ayesh, 2008) proposed the access network selection based on Fuzzy logic and Genetic algorithm. The authors designed a general multicriteria software assistant that can consider user, operator and the QoS view points.

In the proposed work, the input parameters considered for selection are signal strength, speed of the mobile terminal and network coverage of two access networks and Quality of Service parameters. In this work, network coverage of both networks is considered for the selection of best RAT. This study uses fuzzy logic controllers and multi-objective decision making algorithm employing weighting function and Particle Swarm Optimization algorithm for the selection of RAT. The proposed framework is explained in Fig. 2. In this diagram, the input parameters of WWAN and WLAN are fed into the fuzzy logic controllers. The output of the individual fuzzy logic controllers are then fed into the multi objective decision making algorithm to decide the best Radio access technology network among WWAN and WLAN.
Fuzzy logic controller for received signal strength from different networks: The input variables are obtained by measuring the reception signal level of access networks. The first part of the selection algorithm is an array of fuzzy logic controllers. The inputs to the first fuzzy logic controller are signal strength of WWAN and signal strength of WLAN. Here mamdami inference system is considered for the fuzzy controller and centroid method is used for defuzzification. The input has two inputs (SL1 and SL2) where SL1 gives the received signal strength of WWAN and SL2 gives the received signal strength of WLAN and two outputs (SLC1 and SLC2) where SLC1 describes the probability of acceptance for the new user in the WWAN network and SLC2 to describe the probability of acceptance for the new user in the WLAN network. The input variable SL1 starts with the lowest level $(-105 \mathrm{dBm})$ ends with $(-70 \mathrm{dBm})$. The input variable SL2 has the range $(-105 \mathrm{dBm}$ to -60 $\mathrm{dBm})$. The variables SL1 and SL2 have 3 fuzzy sets namely low, medium and high.

The inputs are fed into the fuzzy controller. SLC1 and SLC2 have 4 fuzzy sets namely: Y (Yes), PY (Probably Yes), PN (Probably No) and N (No). By using 9 fuzzy rules, crisp quantities are fuzzified and again defuzzified as crisp values.

Fuzzy logic controller for speed of the mobile terminal: The input variable results are obtained by measuring the speed of the terminal carried by the user at the point of required service. The inputs to the second fuzzy logic controller are speed of the mobile terminal. The speed of the mobile terminal decides the selection of WWAN or WLAN. The linguistic variable speed has 3 fuzzy sets: low, medium and high. The range of the variable speed is set between $0-10 \mathrm{~km} / \mathrm{h}$.

The system has one input (TV) and two outputs (TVC1 and TVC2) related to the access networks of WWAN and WLAN. The inputs are fed into the fuzzy controller and outputs are obtained. When the speed of the mobile terminal is low, then the probability of selection of WLAN will be larger and if the speed is high, the probability of selection of WWAN will be larger.

Fuzzy logic controller for network coverage of radio access technology networks: This system has two inputs ( $\mathrm{NC1}$ and $\mathrm{NC} 2)$ where $\mathrm{NC} 1$ describes the network coverage of WWAN and NC2 describes the network coverage of WLAN and has two outputs TSC1 and TSC2 which represents the probability of selection of WWAN and WLAN. The input variable NC1 and NC2 have the range between 0-10. The input variables have 3 fuzzy sets: bad, medium and good and output variable have 4 fuzzy sets namely Y (Yes), PY (Probably Yes), PN (Probably No) and N (No). 


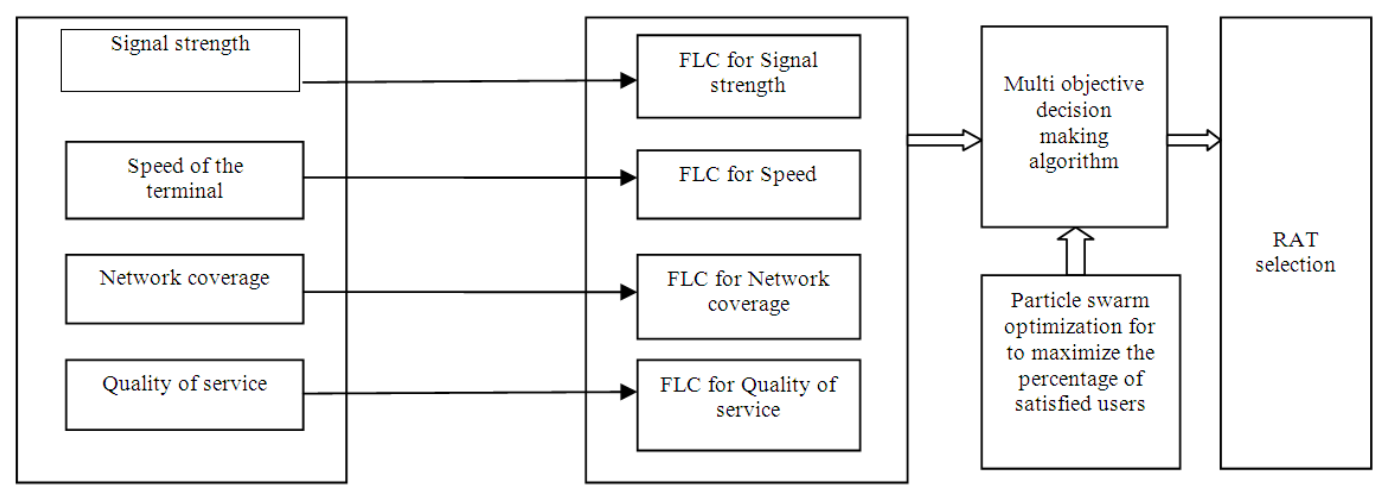

Fig. 2: The proposed architecture of RAT selection

When the network coverage of WWAN is good and network coverage of WLAN is bad then the probability of selection of WWAN will be larger and when the network coverage of WLAN is good and network coverage of WWAN is bad then the probability of selection of WLAN will be larger.

Fuzzy logic controller for Quality of Service: Quality of Service depends on the two paramerters packet delay and packet bit rate. This system has two inputs (QoS1 and QoS2) where QoS1 describes the delay of packets and QoS2 describes the packet bit rate and it has two outputs namely (QoSC1 and QoSC2) which represents the probability of selection of WWAN and WLAN. The delay ranges between 0 to $1000 \mathrm{~ms}$ and bit rate ranges between 0 to $350 \mathrm{~kb} / \mathrm{s}$. By decreasing the value of delay and by reducing the value of bit rate, the probabilty of selection of WWAN will be larger. It is also concluded that by increasing the value of delay and by increasing the value of bit rate, the probability of selection of WLAN will be larger.

Algorithm: The Proposed Hybrid Multi Objective Decision Making Algorithm employing Weighing function and Particle Swarm Optimization.

The main objective of this algorithm is to perform the best selection of access network and to maximize the percentage of the satisfied users. It is important to design a suitable decision making algorithm for the selection of best access network among WWAN and WLAN. In this algorithm, a new weighing function is introduced and Particle Swarm Optimization is used for the maximizing the percentage of satisfied users. The proposed multi-objective decision making algorithm employing Weighing function and Particle Swarm Optimization algorithm is as follows:

Step1: Input the results obtained from the evaluation of input variables through fuzzy logic systems for the given input criteria: SLC1, SLC2, TVC1, TVC2, TSC1, TSC2, QoSC1 and QoSC2.

Step2: Call Particle Swarm Optimization algorithm PSO with input arguments SLC1, SLC2, TVC1, TVC2, TSC1, TSC2, QoSC1 and QoSC2. The output from PSO is the percentage of satisfied users $(\varepsilon)$ as explained in Eq. 1:

$\varepsilon=\mathrm{PSO}(\mathrm{SLC} 1, \mathrm{SLC} 2, \mathrm{TVC} 1, \mathrm{TVC} 2$,

TSC1, TSC2, QoSC1, QoSC2)

Step3: Comparison is made between WWAN and WLAN using IF-THEN condition to select the best cell.

If WWAN >WLAN

Then WWAN is selected

Else if WWAN==WWAN

Then WWAN/WLAN can be selected

Else WWAN $<$ WLAN

Then WLAN is selected

End

Step4: Stop the process when computation is done for all the number of users and the value $(\varepsilon)$ gives the percentage of satisfied users $\left(\mathrm{P}_{\mathrm{u}}\right)$.

Particle swarm optimization: The Particle Swarm Optimization (PSO) first introduced by Kenedy and Eberhart (1995) and Shi and Eberhart (1999).

The basic idea of PSO is as follows: the population is called a swarm and the individuals (i.e. the search points) are called particles. Each particle represents a possible solution to the optimization task at hand. Each particle moves during each iteration with an adaptable velocity within the search space and retains a memory of the best position it ever encountered. In the global version of the PSO, the particle is attracted towards the location of the best fitness achieved so far by the 
particle itself and by the location of the best fitness achieved so far across the whole swarm.

A particle has the following information to make a suitable change in its position and velocity:

- A global best that is known to all and immediately updated when a new best position is found by any particle in the swarm (gbest)

- The local best, which is the best solution that the particle has seen (pbest)

Velocity update Eq. 2 is given by:

$\mathrm{V}_{\mathrm{n}+1}=\mathrm{V}_{\mathrm{n}}+\mathrm{C} 1 * \mathrm{r} 1 *\left(\mathrm{P}_{\text {best }, \mathrm{n}}\right.$

-Current position[n]) +

$\mathrm{C} 2 * \mathrm{r} 2 *\left(\mathrm{G}_{\text {best }, \mathrm{n}}-\right.$ Current position[n] $)$

Using Eq. 3, a certain velocity that gradually gets close to pbests and gbest can be calculated. The current position (searching point in the solution space) can be modified by the following equation:

CurrentPosition $[\mathrm{n}+1]=$

CurrentPosition $[\mathrm{n}]+\mathrm{V}_{[\mathrm{n}+1]}$

Where:

$\mathrm{V}_{\mathrm{n}+1}$ : Velocity of particle at $\mathrm{n}+1$ th iteration

$\mathrm{V}_{\mathrm{n}}$ : Velocity of particle at nth iteration

$\mathrm{C} 1$ : acceleration factor related to pbest

$\mathrm{C} 2$ : acceleration factor related to gbest

$\mathrm{r} 1$ : random number between 0 and 1

r2: random number between 0 and 1

$\mathrm{G}_{\text {best, } \mathrm{n}}$ : gbest position of swarm

$\mathrm{P}_{\text {best,n: }}$ pbest position of particle

Current Position [n+1]: position of particle at $n+1$ th iteration

Current Position[n]: position of particle at nth iteration

$\mathrm{V}_{[\mathrm{n}+1]}$ : particle velocity at $\mathrm{n}+1$ th iteration

Algorithm: particle swarm optimization (SLC1, SLC2, TVC1, TVC2, TSC1, TSC2, QoSC1, QoSC2):

This algorithm takes as input argument the outputs from four fuzzy logic controllers and gives the probability of selection of satisfied users (SS).This algorithm is as follows:

Step1: Select the number of particles, generations, tuning accelerating coefficients $\mathrm{C} 1, \mathrm{C} 2$ and random numbers $\mathrm{r} 1, \mathrm{r} 2$, to start the optimal solution searching.

Step2: Initialize the particle position with the parameters SLC1, SLC2, TVC1, TVC2, TSC1, TSC2, QoSC1 and QoSC2.
Step3: Evaluate the fitness function The weighing function Net1 as given in Eq. 4 is got by summing the outputs of WWAN selection of 4 fuzzy logic controllers and dividing it by the total number of input parameters.

Net $1=\frac{\mathrm{SLC} 1+\mathrm{TVC} 1+\mathrm{TSC} 1+\mathrm{QoS} 1}{\text { total number of input parameters }}$

Similarly, the weighing function Net2 for WLAN as given in Eq. 5 is got by summing the outputs of WLAN selection of 4 fuzzy logic controllers and dividing it by the total number of input parameters:

$\mathrm{Net} 2=\frac{\mathrm{SLC} 2+\mathrm{TVC} 2+\mathrm{TSC} 2+\mathrm{QoS} 2}{\text { total number of input parameters }}$

If the Received Signal strength of WWAN > Received Signal Strength of WLAN, then the Eq. 6 is followed else Eq. 7 is followed:

$\mathrm{Z}=\mathrm{Net} 1 / \mathrm{Net} 2$

Else:

$\mathrm{Z}=\mathrm{Net} 2 / \mathrm{Net} 1$

End

If $Z>1$ then the no. of satisfied users $(\varepsilon)$ in Eq. 8 as given below is taken as the objective function for the particle swarm optimization.

$\varepsilon=\varepsilon+1$

Step4: Select particles individual best value for each generation. Select the particles global best value, i.e. particle near to the target among all the particles is obtained by comparing all the individual best values.

Step5: Update particle individual best ( $\mathrm{p}$ best), global best (g best), in the velocity Eq. 2 and obtain the new velocity

Step6: Update new velocity value from the Eq. 3 and obtain the new position of the particle

Step7: Find the optimal solution by the updated new velocity and position and return the number of satisfied users to the previous algorithm.

\section{RESULTS}

This section presents the details of the simulation carried out on the datasets randomly created to demonstrate the proposed work for the selection of the radio access network. This study is developed in MATLAB environment. 


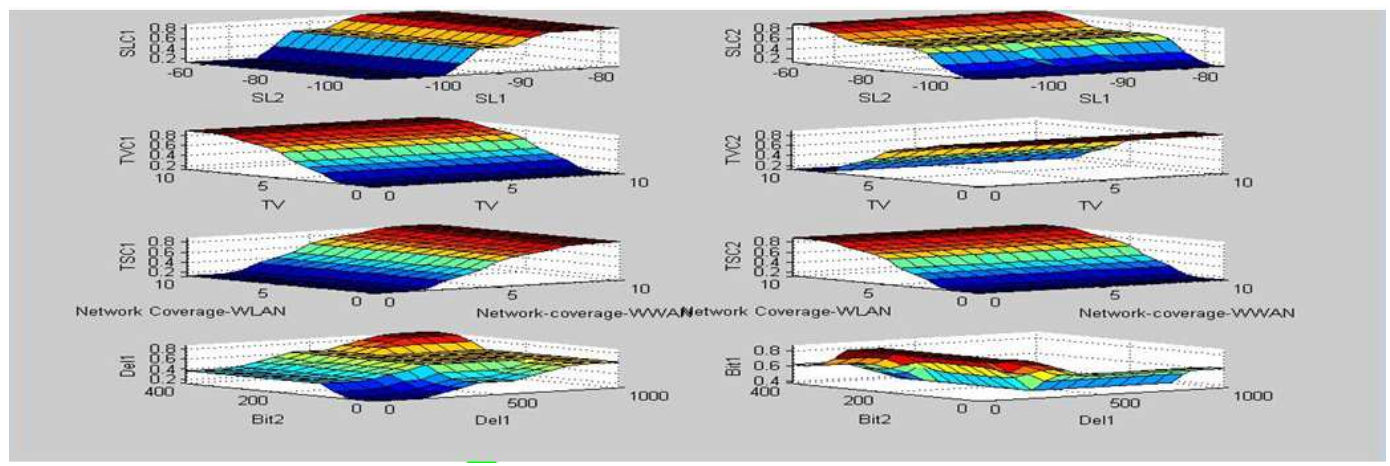

Fig. 3: Outputs from 4 fuzzy logic controllers

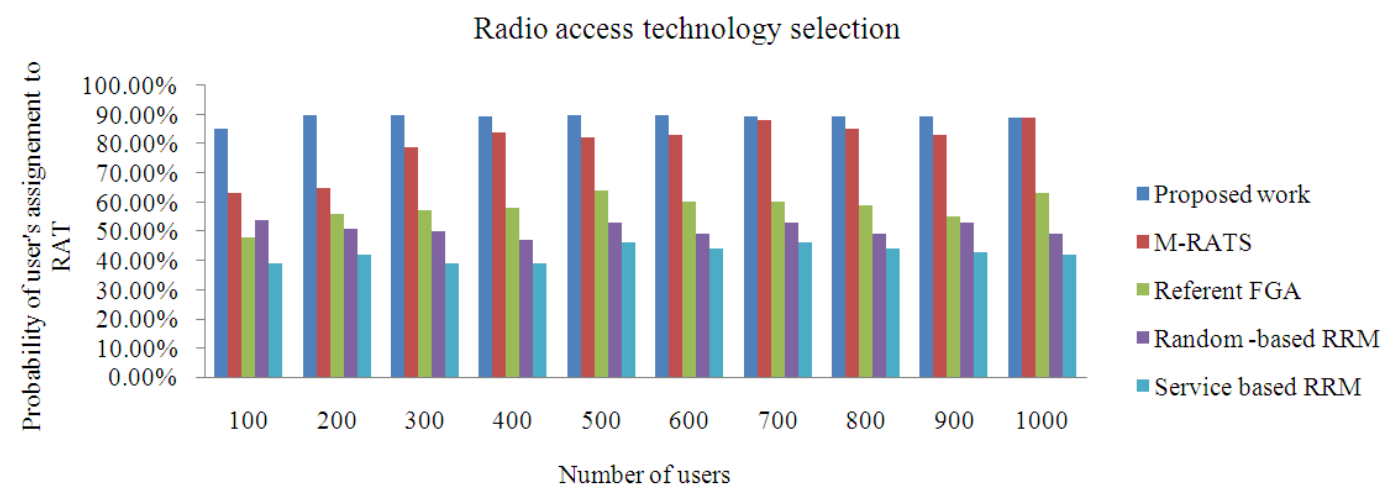

Fig. 4: Chart for Radio Access Technology selection with different number of users

The details of the simulation and the performance of the proposed system are presented here. Two access points considered for selection which are WLAN and WWAN. Users have to select the best access network between these two networks. A dataset consisting of 1000 users each having the signal strength, speed and network coverage of these networks and Quality of Service is considered. This work was carried out in Intel core Duo processors with $2.26 \mathrm{GHz}$ speed and 2GB RAM.

The Fig. 3 shows the graph obtained from the 4 fuzzy logic controllers signal strength, speed of the mobile terminal and network coverage of the networks and QoS. In Fig. 3, the first two graphs concludes that when the value of SL1 (signal strength of WWAN) is increased, the probability of selection of WWAN is larger and when the value of SL2 is increased, the probability of selection of WLAN is larger.

The next two graphs concludes that by increasing the speed of the mobile terminal, WWAN network is selected and by the decreasing the speed, WLAN network is selected. The next two graphs concludes that by increasing the network coverage of WWAN, the probability of selection of WWAN is higher and by increasing the network coverage of WLAN, the probability of selection of WLAN is higher. The last two graphs says that by decreasing the delay and by decreasing the bit rate, the probability of selection of WLAN is larger and by increasing the value of delay and by increasing the value of bit rate, the probability of selection of WWAN is larger.

\section{DISCUSSION}

This section discusses about the simulation results and compares the performance of the proposed work with other four different algorithms. Figure 4 shows the comparison of the proposed algorithm with others. In this, the first algorithm is M-RATS (Mobile based Radio Access Technology

Selection) as proposed by the authors (Aleksandar and Janevski, 2011) where Particle swarm optimization, genetic algorithm and multi criteria decision making algorithm for the selection of RAT. The second algorithm is referent FGA and third algorithm is random based RRM where the users are assigned randomly to the two networks and fourth algorithm is service-based RRM. Figure 4 shows $\mathrm{P}_{\mathrm{u}}$ values. The 
horizontal axis shows the number of users while vertical axis shows the $\mathrm{P}_{\mathrm{u}}$ values. From this chart, it is understood that there is great improvement in the number of the satisfied users in the proposed solution can be seen.

With less number of users (e.g., 100 or 200 users) in the scenario, the proposed work shows better behavior from well known algorithms including Referent FGA, Random based RRM Service based RRM and M-RATs. The results pertaining to the probability of assignment of users to the best RAT was between $85-90 \%$ for all the number of users. Thus, by using the proposed multi-objective decision making algorithm for heterogeneous networks, best RAT can be selected for the user.

\section{CONCLUSION}

In heterogeneous wireless networks, different RATs coexist in the same coverage area. The goal is to select the most suitable RAT for each user. In this study, a multi objective decision making algorithm employing weighing function and Particle swarm optimization based on fuzzy controller has been developed and applied to Radio access Technology selection. The proposed work has been compared against other well known algorithms. From the results, it is observed that the proposed work gives better results than the other algorithms. The future work in this area is to determine the best access technology among the available RATs by giving priority levels among the different classes of calls namely new calls, horizontal handoff calls and vertical handoff calls in heterogeneous wireless networks.

\section{REFERENCES}

Agusti, R., O. Sallent, J. Pérez-Romero and L. Giupponi, 2004. A fuzzy-neural based approach for joint radio resource management in a beyond $3 \mathrm{G}$ framework. Proceedings of the 1st International Conference on Quality of Service in Heterogeneous Wired/Wireless Networks, QSHINE, Oct. 18-20, IEEE Xplore Press, pp: 216-224. DOI: 10.1109/QSHINE.2004.3

Agusti, G.L., R. Perez-Romero and J.O. Sallent Roig, 2008. A novel approach for joint radio resource management based on fuzzy neural methodology. IEEE Trans. Vehicular Technol., 57: 1789-1805. DOI: $10.1109 /$ TVT.2007.907012

AI-Odienat, A.I. and A.A. Al-Lawama, 2008. The advantages of PID fuzzy controllers over the conventional types. Am. J. Applied Sci., 5: 653658. DOI: 10.3844/ajassp.2008.653.658
Aleksandar, T. and T. Janevski, 2011. Efficient radio access technology selection for the next generation wireless networks. Int. J. Res. Rev. Next Generat. Netw., 1: 14-25.

Alkhawlani, M. and A. Ayesh, 2008. Access network selection based on fuzzy logic and genetic algorithms. Adv. Artif. Intell., 08: 1-12. DOI: $10.1155 / 2008 / 793058$

Asif, S.Z., 2007. Wireless Communications: Evolution to $3 \mathrm{G}$ and Beyond. 1st Edn., Artech House, Boston, ISBN-10: 1596930225, pp: 281.

Falowo, O.E. and H.A. Chana, 2008. Joint call admission control algorithms: Requirements, approaches and design considerations. Com. Commun., 31: 1200-1217. DOI: 10.1016/j.comcom.2007.10.044

Hassan, S.S. and R. Hassan, 2011. IPV6 network mobility route optimization survey. Am. J. Applied Sci., $\quad 8$ : 579-583. DOI: 10.3844/ajassp.2011.579.583

Kenedy, J. and R. Eberhart, 1995. Particle swarm optimization. proc. Proceedings of the IEEE International Conference on Neural Networks, Nov. 27-Dec. 1, IEEE Xplore Press, Perth, WA, Australia, pp: 1942-1948. DOI: 10.1109/ICNN.1995.488968

Perez-Romero, J., O. Sallent and R. Agusti, 2005. Policy-based Initial RAT selection algorithms in Heterogeneous Networks. Universitat Politecnica de Catalunya (UPC), Spain.

Perez-Romero, J., O. Sallent and R. Agusti, 2006. Enhanced radio access technology selection exploiting path loss information. Proceedings of the IEEE 17th International Symposium on Personal, Indoor and Mobile Radio Communication, Sept. 11-14, IEEE Xplore Press, Helsinki, pp: 1-5. DOI: 10.1109/PIMRC.2006.254198

Ross, T.J., 2010. Fuzzy Logic with Engineering Applications. 1st Edn., John Wiley and Sons, Chichester, ISBN: 9780470748510 , pp: 585.

Sabbagh, A.L., R. Braun and M. Abolhasan, 2011. A Comprehensive survey on RAT selection algorithms for heterogeneous networks. World Acad. Sci., Eng. Technol., 73: 141-145.

Shi, Y. and R.C. Eberhart, 1999. Empirical study of particle swarm optimization. Proceeding of the 1999 IEEE Congress on Evolutionary Computation, Jul. 6-9, IEEE Xplore Press, Washington, DC, USA., pp: 1945-1950. DOI: 10.1109/CEC.1999.785511

Sivanandam, S.N. and S.N. Deepa, 2007. Principles of Soft Computing. 1st Edn., Wiley India, New Delhi, ISBN: 9788126510757, pp: 762. 
Suleiman, K.H., H.A. Chan and M.E. Dlodlo, 2006. Load balancing in the call admission control of heterogeneous wireless networks. Proceedings of the International Conference on Communications and Mobile computing Vancouver, Jul. 3-6, British Columbia, Canada, pp: 245-250. DOI: $10.1145 / 1143549.1143599$

Takagi, T. and M. Sugeno, 1985. Fuzzy Identification of systems and its applications to modeling and control. IEEE Trans. Syst. Man Cybern., 15: 116-132.

Tolli, A. and P. Hakalin, 2002. Adaptive load balancing between multiple cell layers. Proceedings of the 2002 IEEE 56th Vehicular Technology Conference, (VTC' 02), IEEE Xplore Press, pp: 1691-1695.

DOI:
Umbert, A., L. Budzisz, N. Vucevic and F. Bernardo, 2007. An all-IP heterogeneous wireless testbed for RAT selection and e2e QoS evaluation. Proceedings of the International Conference on Next Generation Mobile Applications, Services and Technologies (NGMAST), Sept. 12-14, IEEE Xplore Press, Cardiff, pp: 310-315. DOI: 10.1109/NGMAST.2007.4343438

Yuhui, S., E. Russell and C. Yaobin, 1999. Implementation of evolutionary fuzzy systems. IEEE Trans. Fuzzy Syst., 7: 109-119.

Zhang, W., 2005. Performance of real-time and data traffic in heterogeneous overlay wireless networks. University of Stuttgart, Stuttgart. 